\title{
Error Analysis of Fixed-Gain AF Relaying with MRC over Nakagami- $m$ Fading Channels
}

\author{
Sajid H. ALVI ${ }^{1,2}$, Shurjeel WYNE ${ }^{1}$ \\ ${ }^{1}$ Dept. of Electrical Engineering, COMSATS Institute of Information Technology, Islamabad, Pakistan \\ ${ }^{2}$ Dept. of Physics, COMSATS Institute of Information Technology, Islamabad, Pakistan
}

\{sajid_hussain, shurjeel.wyne\}@ comsats.edu.pk.

Manuscript received April 28, 2015

\begin{abstract}
This article investigates the error performance of wireless communication systems that employ binary modulations and Amplify-and-Forward $(A F)$ relaying over flat Nakagami-m faded links with maximum ratio combining (MRC) at destination. Specifically, we derive a simple yet accurate closed-form approximation for the average bit error probability $(A B E P)$ and closed-form expressions for its tight upper and lower bounds. The effect of power imbalance between the relayed links is also studied. Numerical investigations show good agreement between proposed theoretical results and simulations whereas our performance bounds are shown to be tighter than previously proposed bounds for the case of unbalanced relayed links.
\end{abstract}

\section{Keywords}

Amplify-and-forward (AF) relaying, maximal-ratio combining (MRC), Nakagami fading, error analysis.

\section{Introduction}

Cooperative relaying is an efficient strategy to combat fading and to increase communication reliability of wireless communication links [1]. Relays can be deployed to enhance coverage and capacity near the cell edges and beyond in cellular networks [2]. The Amplify-and-Forward (AF) relay simply amplifies the received signal before transmitting it to the destination. It thus provides a low complexity low cost solution relative to the regenerative relays albeit at the expense of undesirable noise amplification [3]. The AF relaying schemes can be further classified as variable gain and fixed gain/semi-blind relaying. The former mode uses the instantaneous channel state information (CSI) of the previous hop to control the relay gain whereas the latter uses channel statistics of the previous hop to determine relay gain. The fixed gain AF (FG-AF) relaying scheme trades off the diversity performance of variable gain schemes with lower complexity in the CSI estimation and are therefore more attractive due to practical considerations [4].

Cooperative communications over Nakagami- $m$ faded links with and without diversity combining have been ex- tensively studied in the literature, see for example [5-8] and references therein. In [5] the authors consider a dual-hop FGAF link without diversity and Nakagami-m faded channels with arbitrary values of $m$; under these conditions they derive closed-form expressions for the ABEP of binary and quaternary phase shift keying (PSK). In [6] the authors also consider a dual-hop FG-AF link without diversity and the Nakagami$m$ faded channels are considered to take arbitrary values of $m$; under these conditions they derive a closed-form approximation for the average symbol error probability (ASEP) for M-ary phase shift keying (MPSK). In [7] FG-AF relaying with MRC diversity at destination and Nakagami- $m$ fading with arbitrary $m$ is considered; the authors derive an exact expression of the ABEP for MPSK but it requires numerical evaluation of the moment generating function (MGF) of the instantaneous signal-to-noise ratio (SNR) at MRC output. The authors in [8] consider a multi-relay system with FG-AF relaying and MRC diversity at destination. Channel fading is assumed to be independent but identically-distributed Nakagami on all links with the same integer-valued fading parameter $m$ on all links. Under these fading conditions the authors in [8] derive upper and lower bounds on the ASEP for MPSK, which require numerical integration for their evaluation.

The submitted work considers a single relay system with FG-AF relaying and MRC diversity at destination, which operates over independent but not necessarily identically distributed Nakagami- $m$ faded channels. We derive a simple yet accurate closed-form approximation for the ABEP of coherently detected binary phase shift keying (BPSK) and binary frequency shift keying (BFSK). It may be noted that many works have focused on the ABEP analysis of only one modulation scheme such as coherently detected BPSK in [3], [9], [10] or $\frac{\pi}{4}$-Differential Quaternary phase shift keying (QPSK) in [11]. Our analysis is more general in that it applies to both BPSK and BFSK. Furthermore, since coherently detected BPSK and QPSK have the same ABEP [12], our derived results are also applicable to QPSK. Our proposed analytical approach is independent of the approach used by [7] to derive the exact ABEP expressions and it yields an expression that is equally accurate but much simpler to evaluate 


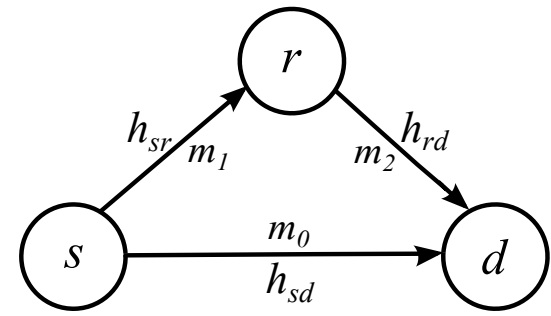

Fig. 1. Cooperative diversity network under consideration.

than the exact expression of [7]. We also propose analytic closed-form expressions for tight upper and lower bounds on the ABEP for the same system and channel conditions. The proposed bounds are easier to evaluate than the bounds of [8] and at the same time they do not have the restriction to consider only identically distributed Nakagami- $m$ faded links as observed in the analysis of [8]. The proposed expressions are validated through simulations for various values of the Nakagami fading parameter $m$ and are shown to outperform the results given in previous work, while being considerably simpler to evaluate than the latter.

The remainder of this letter is organized as follows. The system and channel model under consideration are introduced in Section 2 and details of analytical expressions are provided in Section 3. Results are discussed in Section 4. Finally, some concluding remarks are given in Section 5.

\section{System and Channel Model}

We consider the cooperative diversity network shown in Fig. 1 in which nodes transmit over orthogonal channels. In the first time slot, the source transmits directly to the destination and single relay. In the second time slot the relay amplifies its received signal and transmits it to the destination, which combines the two received signals using MRC. Let $h_{s d}, h_{s r}$ and $h_{r d}$ denote the independent flat-fading Nakagami- $m$ channel coefficients with corresponding fading parameters $m_{0}, m_{1}$, and $m_{2}$ for the source-destination (s-d), source-relay (s-r), and relay-destination (r-d) links, respectively. It follows that the instantaneous channel power gains $\left|h_{i j}\right|^{2}, i, j \in(s, r, d)$ are Gamma distributed random variables [7] with corresponding average values denoted by $\sigma_{i j}^{2}, i, j \in(s, r, d)$. Using MRC at destination, the instantaneous SNR at the combiner output can be written as [3]

$$
\gamma_{c}=\gamma_{0}+\frac{\gamma_{1} \gamma_{2}}{\gamma_{2}+C_{c}}=\gamma_{0}+\gamma_{r}
$$

where $\gamma_{0}, \gamma_{1}$ and $\gamma_{2}$ are the instantaneous SNRs with corresponding average values $\bar{\gamma}_{0}=\frac{E_{s}}{N_{0}} \sigma_{s d}^{2}, \bar{\gamma}_{1}=\frac{E_{s}}{N_{0}} \sigma_{s r}^{2}$ and $\bar{\gamma}_{2}=\frac{E_{s}}{N_{0}} \sigma_{r d}^{2}$ for the s-d, s-r, and r-d links, respectively. We have assumed that $\sigma_{s d}^{2}=1$ without loss of generality. $E_{s}$ is the identical average transmit power at source and relay, whereas $N_{o}$ is the identical power spectral density of additive white Gaussian thermal noise present at all node inputs. The constant $C_{C}$ in (1) depends on the type of fixed-gain relaying and for the relay power gain factor chosen as [7], [4]

$$
G^{2}=E\left\{\frac{E_{s}}{E_{s}\left|h_{s r}\right|^{2}+N_{0}}\right\}
$$

where $E$ denotes the statistical expectation operator, $C_{c}$ can be written as [7]

$$
C_{c}=\left[\frac{m_{1}}{\bar{\gamma}_{1}} \psi\left(1,2-m_{1} ; \frac{m_{1}}{\bar{\gamma}_{1}}\right)\right]^{-1}
$$

where $\psi($.$) denotes the confluent hyper geometric func-$ tion [13]. Now using the functional relation between the hyper geometric function and the incomplete gamma function, $C_{c}$ can be written as [13]

$$
C_{c}=\frac{1}{\left(\frac{m_{1}}{\bar{\gamma}_{1}}\right)^{m_{1}} e^{\frac{m_{1}}{\bar{\gamma}_{1}}} \Gamma\left(1-m_{1}, \frac{m_{1}}{\bar{\gamma}_{1}}\right)}
$$

where $\Gamma(a, x)$ is the incomplete gamma function [13].

\section{Error Analysis}

A generic expression for the ABEP for coherently detected BPSK and BFSK can be written as [14], [15]

$$
\bar{P}_{\mathrm{b}}=E\left[\frac{\Gamma\left(\frac{1}{2}, a \gamma_{c}\right)}{2 \Gamma\left(\frac{1}{2}\right)}\right]=E\left[\frac{\operatorname{erfc}\left(\sqrt{a \gamma_{c}}\right)}{2}\right]
$$

where $\bar{P}_{b}$ denotes the expected value of $P_{\mathrm{b}}$, which is the instantaneous bit error probability (BEP), $\Gamma$ (.) is the wellknown gamma function and erfc (.) is the well-known complementary error function. Furthermore, the second equality in (5) above is obtained from [16]. Note that by setting $a=1$ in (5) the ABEP for coherent BPSK and QPSK is obtained [12], whereas using $a=\frac{1}{2}$ in (5) gives the ABEP for coherent BFSK [17]. The presence of erfc $(x)$ in (5) allows us to leverage the rich literature available on its bounds and approximations. For example, [18] presented an upper bound for erfc $(x)$ and its approximation, which also serves as a tight upper-bound for $x>0.5$. In [19] a lower bound for erfc $(x)$ was proposed. Recently, [20] proposed a new approximation for erfc $(x)$, which was shown to be more accurate than the approximation of [18]. We now use these results to derive tight upper and lower bounds as well as a simple yet accurate approximation to the considered ABEP.

Using the erfc $(x)$ upper-bound proposed in [18] and applying the monotonicity property of expectation for two random variables $X \leq Y \Rightarrow E[X] \leq E[Y]$ [21], we obtain an upper-bound on the ABEP in (5) that can be formulated as

$$
\bar{P}_{\mathrm{b}} \leq E\left[P_{\mathrm{b}}^{\mathrm{UB}}\right] \equiv \frac{1}{12} E\left[2 e^{-4 a \gamma_{c}}+e^{-2 a \gamma_{c}}+3 e^{-a \gamma_{c}}\right] .
$$

Similarly using the lower bound on erfc $(x)$ proposed in [19] and applying the monotonicity property of expectation, a lower-bound on the ABEP in (5) can be obtained as

$$
\bar{P}_{\mathrm{b}} \geq E\left[P_{\mathrm{b}}^{\mathrm{LB}}\right] \equiv \frac{1}{12} E\left[e^{-4 a \gamma_{c}}+e^{-2 a \gamma_{c}}+2 e^{-\frac{4}{3} a \gamma_{c}}\right] .
$$


Now to derive a simple yet accurate approximation for the ABEP in (5), the order 3 polynomial approximation of erfc $(x)$ given in [20] is used to obtain

$$
\bar{P}_{\mathrm{b}} \approx E\left[P_{\mathrm{b}}^{\mathrm{aprx}}\right] \equiv \frac{1}{2} E\left[c_{1} e^{-c a \gamma_{c}}+c_{2} e^{-2 c a \gamma_{c}}+c_{3} e^{-3 c a \gamma_{c}}\right]
$$

where $c_{1}=0.3357, c_{2}=0.3361, c_{3}=0.0305$, and $c=1.0649$ are curve fitting coefficients [20]. Noting that $e^{-c a \gamma_{c}}=$ $e^{-c a \gamma_{0}} \times e^{-c a \gamma_{r}}$ and using the statistical independence of $\gamma_{0}$ and $\gamma_{r}$, the expectation can be operated in (6), (7), and (8) to simplify results. First using the series expansion of $e^{-x}$ from [13], $E\left[e^{-c a \gamma_{0}}\right]$ can be written as

$$
E\left[e^{-c a \gamma_{0}}\right]=\sum_{n=0}^{\infty} \frac{(-1)^{n}}{n !} E\left[\left(c a \gamma_{0}\right)^{n}\right]
$$

where the $n^{\text {th }}$ moment of ( $\left.c a \gamma_{0}\right)$ can be written using [22] as

$$
E\left[\left(c a \gamma_{0}\right)^{n}\right]=\frac{\Gamma\left(m_{0}+n\right)}{\Gamma\left(m_{0}\right)}\left(\frac{c a \bar{\gamma}_{0}}{m_{0}}\right)^{n} .
$$

Substituting (10) into (9) and using the Pochhammer's symbol representation $\left(m_{0}\right)_{n}=\frac{\Gamma\left(m_{0}+n\right)}{\Gamma\left(m_{0}\right)}$ [16], we get

$$
\begin{aligned}
E\left[e^{-c a \gamma_{0}}\right] & =\sum_{n=0}^{\infty} \frac{\left(m_{0}\right)_{n}}{n !}\left(-\frac{c a \bar{\gamma}_{0}}{m_{0}}\right)^{n}, \\
& ={ }_{1} F_{0}\left(m_{0} ;-;-\frac{c a \bar{\gamma}_{0}}{m_{0}}\right) .
\end{aligned}
$$

Here, ${ }_{1} F_{0}(\alpha ;-; z)$ is the Confluent Hypergeometric function [13]. All other exponential functions with $\gamma_{0}$ in their exponent can be dealt accordingly.

To evaluate $E\left[e^{-c a \gamma_{r}}\right]$ we exploit the statistical independence of $\gamma_{1}$ and $\gamma_{2}$; then use $e^{-x}=\Gamma(1, x)[13]$ and the series expansion of $\Gamma(1, x)[13]$ to obtain,

$$
\begin{array}{r}
E\left[e^{-c a \gamma_{r}}\right]=1-\sum_{n=0}^{\infty} \frac{(-1)^{n}}{n !(1+n)} E\left[\left(c a \gamma_{1}\right)^{1+n}\right] \\
\times E\left[\left(\frac{\gamma_{2}}{C_{c}+\gamma_{2}}\right)^{1+n}\right] .
\end{array}
$$

Now the $(1+n)^{\text {th }}$ moment of $\left(c a \gamma_{1}\right)$ in (12) can be simplified by using (10) and after employing the Pochhammer's symbol representation of $\Gamma\left(m_{1}+1+n\right),(12)$ simplifies to,

$$
\begin{aligned}
E\left[e^{-c a \gamma_{r}}\right]=1-c a \bar{\gamma}_{1} \sum_{n=0}^{\infty} \frac{\left(m_{1}+1\right)_{n}}{n !(1+n)}\left(-\frac{c a \bar{\gamma}_{1}}{m_{1}}\right)^{n} \\
\times E\left[\left(\frac{\gamma_{2}}{C_{c}+\gamma_{2}}\right)^{1+n}\right] .
\end{aligned}
$$

Let $\gamma_{22} \equiv \frac{\gamma_{2}}{C_{c}+\gamma_{2}}$, then its $(1+n)^{\text {th }}$ moment can be defined using the MGF approach as [14]

$$
E\left[\left(\gamma_{22}\right)^{1+n}\right] \equiv \frac{1}{n !} \int_{0}^{\infty} S^{n} M_{\frac{1}{\gamma_{22}}}(S) d S
$$

where $M_{\frac{1}{\gamma_{22}}}$ (.) is the MGF of $\frac{1}{\gamma_{22}}$ written as [22]

$$
M_{\frac{1}{\gamma_{22}}}(S)=\frac{2\left(\frac{C_{c} m_{2}}{\bar{\gamma}_{2}} S\right)^{\frac{m_{2}}{2}} e^{-S}}{\Gamma\left(m_{2}\right)} K_{m_{2}}\left(2 \sqrt{\frac{C_{c} m_{2}}{\bar{\gamma}_{2}} S}\right)
$$

where $K_{v}$ (.) is the modified Bessel function of second kind and order $v$ [13]. Substituting (15) into (14) we get,

$$
\begin{aligned}
& E\left[\left(\gamma_{22}\right)^{1+n}\right]=\frac{2}{\Gamma\left(m_{2}\right) n !}\left(\frac{C_{c} m_{2}}{\bar{\gamma}_{2}}\right)^{\frac{m_{2}}{2}} \\
& \times \int_{0}^{\infty} S^{\frac{m_{2}}{2}+n} e^{-S} K_{m_{2}}\left(2 \sqrt{\frac{C_{c} m_{2}}{\bar{\gamma}_{2}} S}\right) d S .
\end{aligned}
$$

After substituting (16) into (13) and simplifying we get

$$
\begin{aligned}
E\left[e^{-c a \gamma_{r}}\right] & =1-\frac{\left(2 c a \bar{\gamma}_{1}\right)\left(\frac{C_{c} m_{2}}{\bar{\gamma}_{2}}\right)^{\frac{m_{2}}{2}}}{\Gamma\left(m_{2}\right)} \\
& \times \int_{0}^{\infty}{ }_{1} F_{1}\left(1+m_{1} ; 2 ;-\frac{c a \bar{\gamma}_{1} S}{m_{1}}\right) \\
& \times S^{\frac{m_{2}}{2}} e^{-S} K_{m_{2}}\left(2 \sqrt{\frac{C_{c} m_{2}}{\bar{\gamma}_{2}}} S\right) d S
\end{aligned}
$$

where we have used the fact that $(1+n) n !=(1+n) !=$ $(2)_{n} \Gamma(2)$ and employing ${ }_{1} F_{1}(\alpha, \gamma ; z)$ which is the ConfluentHypergeometric function [13]. Now we evaluate the integral part of (17) separately and write it as

$$
\begin{aligned}
\Phi \equiv \int_{0}^{\infty}{ }_{1} F_{1}\left(1+m_{1} ; 2\right. & \left.;-\frac{c a \bar{\gamma}_{1}}{m_{1}} S\right) S^{\frac{m_{2}}{2}} \\
& \times e^{-S} K_{m_{2}}\left(2 \sqrt{\frac{C_{c} m_{2}}{\overline{\gamma_{2}}} S}\right) d S .
\end{aligned}
$$

The integral in (18) seems quite complicated but it can be simplified by considering the relayed-link fading parameter $m_{2}$ to be non-integer ${ }^{1}$. The function $K_{m_{2}}$ (.) can then be written as [23]

$$
\begin{aligned}
K_{m_{2}}\left(2 \sqrt{\frac{C_{c} m_{2}}{\bar{\gamma}_{2}} S}\right) & =\frac{\Gamma\left(-m_{2}\right)}{2}\left(\frac{C_{c} m_{2}}{\bar{\gamma}_{2}} S\right)^{\frac{m_{2}}{2}} \\
& \times{ }_{0} F_{1}\left(-; 1+m_{2} ; \frac{C_{c} m_{2} S}{\bar{\gamma}_{2}}\right) \\
& +\frac{\Gamma\left(m_{2}\right)}{2}\left(\frac{C_{c} m_{2}}{\bar{\gamma}_{2}} S\right)^{-\frac{m_{2}}{2}} \\
& \times{ }_{0} F_{1}\left(-; 1-m_{2} ; \frac{C_{c} m_{2} S}{\bar{\gamma}_{2}}\right)
\end{aligned}
$$

\footnotetext{
${ }^{1}$ The case of integer $m_{2}$ can be treated without loss of performance as $m_{2}+\delta$ for sufficiently small $\delta$. For example, setting $\delta=.01$ admits convergent analytical expressions while channel fading on the r-d link remains practically unchanged. Numerical results shown in the next section also validate this approach.
} 
where ${ }_{0} F_{1}(-; \beta ; z)$ is the Confluent Hypergeometric function whose series expansion [13] simplifies (19) as

$$
\begin{aligned}
K_{m_{2}}\left(2 \sqrt{\frac{C_{c} m_{2}}{\bar{\gamma}_{2}} S}\right) & =\frac{\Gamma\left(-m_{2}\right)}{2}\left(\frac{C_{c} m_{2}}{\bar{\gamma}_{2}} S\right)^{\frac{m_{2}}{2}} \\
& \times \sum_{n=0}^{\infty} \frac{1}{n !\left(1+m_{2}\right)_{n}}\left(\frac{C_{c} m_{2} S}{\bar{\gamma}_{2}}\right)^{n} \\
& +\frac{\Gamma\left(m_{2}\right)}{2}\left(\frac{C_{c} m_{2}}{\bar{\gamma}_{2}} S\right)^{-\frac{m_{2}}{2}} \\
& \times \sum_{n=0}^{\infty} \frac{1}{n !\left(1-m_{2}\right)_{n}}\left(\frac{C_{c} m_{2} S}{\bar{\gamma}_{2}}\right)^{n} .
\end{aligned}
$$

Substituting (20) in (18) and rearranging the terms, we have

$$
\begin{aligned}
\Phi & =\frac{\Gamma\left(m_{2}\right)\left(\frac{C_{c} m_{2}}{\bar{\gamma}_{2}}\right)^{-\frac{m_{2}}{2}}}{2} \sum_{n=0}^{\infty} \frac{1}{n !}\left(\frac{C_{c} m_{2}}{\bar{\gamma}_{2}}\right)^{n}\left\{\frac{\Gamma\left(-m_{2}\right)\left(\frac{C_{c} m_{2}}{\bar{\gamma}_{2}}\right)^{m_{2}}}{\Gamma\left(m_{2}\right)\left(1+m_{2}\right)_{n}}\right. \\
& \times \int_{0}^{\infty} S^{m_{2}+n} e^{-S}{ }_{1} F_{1}\left(1+m_{1} ; 2 ;-\frac{c a \bar{\gamma}_{1}}{m_{1}} S\right) d S \\
& \left.+\frac{1}{\left(1-m_{2}\right)_{n}} \int_{0}^{\infty} S^{n} e^{-S}{ }_{1} F_{1}\left(1+m_{1} ; 2 ;-\frac{c a \bar{\gamma}_{1}}{m_{1}} S\right) d S\right\} .
\end{aligned}
$$

The integrals in (21) can be evaluated using [13] to obtain

$$
\begin{aligned}
\Phi & =\frac{\Gamma\left(m_{2}\right)\left(\frac{C_{c} m_{2}}{\bar{\gamma}_{2}}\right)^{-\frac{m_{2}}{2}}}{2} \sum_{n=0}^{\infty}\left(\frac{C_{c} m_{2}}{\bar{\gamma}_{2}}\right)^{n}\left\{\frac{\Gamma\left(-m_{2}\right) \Gamma\left(1+m_{2}+n\right)}{n ! \Gamma\left(m_{2}\right)\left(1+m_{2}\right)_{n}}\right. \\
& \times\left(\frac{C_{c} m_{2}}{\bar{\gamma}_{2}}\right)^{m_{2}}{ }_{2} F_{1}\left(1+m_{1}, 1+m_{2}+n ; 2 ;-\frac{c a \bar{\gamma}_{1}}{m_{1}}\right) \\
& \left.+\frac{\Gamma(1+n)}{n !\left(1-m_{2}\right)_{n}}{ }_{2} F_{1}\left(1+m_{1}, 1+n ; 2 ;-\frac{c a \bar{\gamma}_{1}}{m_{1}}\right)\right\} .
\end{aligned}
$$

Now substituting (22) into (17) we get

$$
\begin{aligned}
E\left[e^{-c a \gamma_{r}}\right] & =1-\left(c a \bar{\gamma}_{1}\right) \sum_{n=0}^{\infty}\left(\frac{C_{c} m_{2}}{\bar{\gamma}_{2}}\right)^{n}\left\{\frac{\Gamma\left(-m_{2}\right) \Gamma\left(1+m_{2}+n\right)}{n ! \Gamma\left(m_{2}\right)\left(1+m_{2}\right)_{n}}\right. \\
& \times\left(\frac{C_{c} m_{2}}{\bar{\gamma}_{2}}\right)^{m_{2}}{ }_{2} F_{1}\left(1+m_{1}, 1+m_{2}+n ; 2 ;-\frac{c a \bar{\gamma}_{1}}{m_{1}}\right) \\
& \left.+\frac{\Gamma(1+n)}{n !\left(1-m_{2}\right)_{n}}{ }_{2} F_{1}\left(1+m_{1}, 1+n ; 2 ;-\frac{c a \bar{\gamma}_{1}}{m_{1}}\right)\right\} .
\end{aligned}
$$

Combining the results of (11) and (23), $E\left[e^{-c a \gamma_{c}}\right]$ can then be expressed as

$$
\begin{aligned}
E\left[e^{-c a \gamma_{c}}\right] & ={ }_{1} F_{0}\left(m_{0} ;-;-\frac{c a \bar{\gamma}_{0}}{m_{0}}\right) \\
& \times\left[1-\left(c a \bar{\gamma}_{1}\right) \sum_{n=0}^{\infty}\left(\frac{C_{c} m_{2}}{\bar{\gamma}_{2}}\right)^{n}\right. \\
& \times\left\{\frac{\Gamma\left(-m_{2}\right) \Gamma\left(1+m_{2}+n\right)}{n ! \Gamma\left(m_{2}\right)\left(1+m_{2}\right)_{n}}\left(\frac{C_{c} m_{2}}{\bar{\gamma}_{2}}\right)^{m_{2}}\right. \\
& \times{ }_{2} F_{1}\left(1+m_{1}, 1+m_{2}+n ; 2 ;-\frac{c a \bar{\gamma}_{1}}{m_{1}}\right) \\
& \left.\left.+\frac{\Gamma(1+n)}{n !\left(1-m_{2}\right)_{n}}{ }_{2} F_{1}\left(1+m_{1}, 1+n ; 2 ;-\frac{c a \bar{\gamma}_{1}}{m_{1}}\right)\right\}\right]
\end{aligned}
$$

Using (24) in (8) the ABEP approximation is obtained as

$$
\begin{aligned}
\bar{P}_{\mathrm{b}} & \approx \frac{c_{1}}{2}{ }_{1} F_{0}\left(m_{0} ;-;-\frac{c a \bar{\gamma}_{0}}{m_{0}}\right) \\
& \times\left[1-\left(c a \bar{\gamma}_{1}\right) \sum_{n=0}^{\infty}\left(\frac{C_{c} m_{2}}{\bar{\gamma}_{2}}\right)^{n}\right. \\
& \times\left\{\frac{\Gamma\left(-m_{2}\right) \Gamma\left(1+m_{2}+n\right)}{n ! \Gamma\left(m_{2}\right)\left(1+m_{2}\right)_{n}}\left(\frac{C_{c} m_{2}}{\bar{\gamma}_{2}}\right)^{m_{2}}\right. \\
& \times{ }_{2} F_{1}\left(1+m_{1}, 1+m_{2}+n ; 2 ;-\frac{c a \bar{\gamma}_{1}}{m_{1}}\right) \\
& \left.\left.+\frac{\Gamma(1+n)}{n !\left(1-m_{2}\right)_{n}}{ }_{2} F_{1}\left(1+m_{1}, 1+n ; 2 ;-\frac{c a \bar{\gamma}_{1}}{m_{1}}\right)\right\}\right] \\
& +\frac{c_{2}}{2}{ }_{1} F_{0}\left(m_{0} ;-;-\frac{2 c a \bar{\gamma}_{0}}{m_{0}}\right) \\
& \times\left[1-\left(2 c a \bar{\gamma}_{1}\right) \sum_{n=0}^{\infty}\left(\frac{C_{c} m_{2}}{\bar{\gamma}_{2}}\right)^{n}\right. \\
& \left.\times\left\{\frac{{ }_{2} F_{1}\left(1+m_{1}, 1+m_{2}+n ; 2 ;-\frac{3 c a \bar{\gamma}_{1}}{m_{1}}\right)}{n ! \Gamma\left(m_{2}\right)\left(1+m_{2}\right)_{n}}{ }_{2} F_{1}\left(1+m_{1}, 1+n ; 2 ;-\frac{3 c a \bar{\gamma}_{1}}{m_{1}}\right)\right\}\right] \\
& \times\left\{\frac{c_{3}\left(-m_{2}\right) \Gamma\left(1+m_{2}+n\right)}{n ! \Gamma\left(m_{2}\right)\left(1+m_{2}\right)_{n}}\left(\frac{C_{c} m_{2}}{\bar{\gamma}_{2}}\right)^{m_{2}}{ }_{1}\left(m_{0} ;-;-\frac{3 c a \bar{\gamma}_{0}}{m_{0}}\right)\right. \\
& \times{ }_{2} F_{1}\left(1+m_{1}, 1+m_{2}+n ; 2 ;-\frac{2 c a \bar{\gamma}_{1}}{m_{1}}\right) \\
& \times(1+n) \\
& \left.\left.\times{ }_{2} F_{1}\left(1+m_{1}, 1+n ; 2 ;-\frac{2 c a \bar{\gamma}_{1}}{m_{1}}\right)\right\}\right]
\end{aligned}
$$

Similarly using (24) in (6) and (7) the closed-form upper and lower-bounds on the ABEP are obtained as (26) and (27), respectively.

\section{Numerical and Simulation Results}

In this section some numerical and simulation results are provided to support the accuracy of the proposed analytical expressions. For plotting purposes, the first 15 terms of the infinite sums in (25)-(27) have been used. All simulations were carried out using the Matlab computational software, whereas the analytical expressions of [7] and [8] were evaluated using the Mathematica software.

In Fig. 2, the proposed ABEP approximation is plotted for the case of SNR-balanced links, i.e., $\bar{\gamma}_{2}=\bar{\gamma}_{1}$. Furthermore, Fig. 2 a considers $m_{2}=0.5$ a non-integer value 


$$
\begin{aligned}
& \bar{P}_{\mathrm{b}} \leq \frac{1}{6}{ }_{1} F_{0}\left(m_{0} ;-;-\frac{4 a \bar{\gamma}_{0}}{m_{0}}\right)\left[1-\left(4 a \bar{\gamma}_{1}\right) \sum_{n=0}^{\infty}\left(\frac{C_{c} m_{2}}{\bar{\gamma}_{2}}\right)^{n}\left\{\frac{\Gamma\left(-m_{2}\right) \Gamma\left(1+m_{2}+n\right)}{n ! \Gamma\left(m_{2}\right)\left(1+m_{2}\right)_{n}}\left(\frac{C_{c} m_{2}}{\bar{\gamma}_{2}}\right)^{m_{2}}{ }_{2} F_{1}\left(1+m_{1}, 1+m_{2}+n ; 2 ;-\frac{4 a \bar{\gamma}_{1}}{m_{1}}\right)\right.\right. \\
& \left.\left.+\frac{\Gamma(1+n)}{n !\left(1-m_{2}\right)_{n}}{ }_{2} F_{1}\left(1+m_{1}, 1+n ; 2 ;-\frac{4 a \bar{\gamma}_{1}}{m_{1}}\right)\right\}\right] \\
& +\frac{1}{12}{ }_{1} F_{0}\left(m_{0} ;-;-\frac{2 a \bar{\gamma}_{0}}{m_{0}}\right)\left[1-\left(2 a \bar{\gamma}_{1}\right) \sum_{n=0}^{\infty}\left(\frac{C_{c} m_{2}}{\bar{\gamma}_{2}}\right)^{n}\left\{\frac{\Gamma\left(-m_{2}\right) \Gamma\left(1+m_{2}+n\right)}{n ! \Gamma\left(m_{2}\right)\left(1+m_{2}\right)_{n}}\left(\frac{C_{c} m_{2}}{\bar{\gamma}_{2}}\right)^{m_{2}}{ }_{2} F_{1}\left(1+m_{1}, 1+m_{2}+n ; 2 ;-\frac{2 a \bar{\gamma}_{1}}{m_{1}}\right)\right.\right. \\
& \left.\left.+\frac{\Gamma(1+n)}{n !\left(1-m_{2}\right)_{n}}{ }_{2} F_{1}\left(1+m_{1}, 1+n ; 2 ;-\frac{2 a \bar{\gamma}_{1}}{m_{1}}\right)\right\}\right] \\
& +\frac{1}{4}{ }_{1} F_{0}\left(m_{0} ;-;-\frac{a \bar{\gamma}_{0}}{m_{0}}\right)\left[1-\left(a \bar{\gamma}_{1}\right) \sum_{n=0}^{\infty}\left(\frac{C_{c} m_{2}}{\bar{\gamma}_{2}}\right)^{n}\left\{\frac{\Gamma\left(-m_{2}\right) \Gamma\left(1+m_{2}+n\right)}{n ! \Gamma\left(m_{2}\right)\left(1+m_{2}\right)_{n}}\left(\frac{C_{c} m_{2}}{\bar{\gamma}_{2}}\right)^{m_{2}}{ }_{2} F_{1}\left(1+m_{1}, 1+m_{2}+n ; 2 ;-\frac{a \bar{\gamma}_{1}}{m_{1}}\right)\right.\right. \\
& \left.\left.+\frac{\Gamma(1+n)}{n !\left(1-m_{2}\right)_{n}}{ }_{2} F_{1}\left(1+m_{1}, 1+n ; 2 ;-\frac{a \bar{\gamma}_{1}}{m_{1}}\right)\right\}\right] \text {. }
\end{aligned}
$$

$$
\begin{aligned}
& \bar{P}_{\mathrm{b}} \geq \frac{1}{12}{ }_{1} F_{0}\left(m_{0} ;-;-\frac{4 a \bar{\gamma}_{0}}{m_{0}}\right)[1-\left(4 a \bar{\gamma}_{1}\right) \sum_{n=0}^{\infty}\left(\frac{C_{c} m_{2}}{\bar{\gamma}_{2}}\right)^{n}\left\{\frac{\Gamma\left(-m_{2}\right) \Gamma\left(1+m_{2}+n\right)}{n ! \Gamma\left(m_{2}\right)\left(1+m_{2}\right)_{n}}\left(\frac{C_{c} m_{2}}{\bar{\gamma}_{2}}\right)^{m_{2}}{ }_{2} F_{1}\left(1+m_{1}, 1+m_{2}+n ; 2 ;-\frac{4 a \bar{\gamma}_{1}}{m_{1}}\right)\right. \\
&\left.\left.+\frac{\Gamma(1+n)}{n !\left(1-m_{2}\right)_{n}}{ }_{2} F_{1}\left(1+m_{1}, 1+n ; 2 ;-\frac{4 a \bar{\gamma}_{1}}{m_{1}}\right)\right\}\right] \\
&+\frac{1}{12}{ }_{1} F_{0}\left(m_{0} ;-;-\frac{2 a \bar{\gamma}_{0}}{m_{0}}\right)\left[1-\left(2 a \bar{\gamma}_{1}\right) \sum_{n=0}^{\infty}\left(\frac{C_{c} m_{2}}{\bar{\gamma}_{2}}\right)^{n}\left\{\frac{\Gamma\left(-m_{2}\right) \Gamma\left(1+m_{2}+n\right)}{n ! \Gamma\left(m_{2}\right)\left(1+m_{2}\right)_{n}}\left(\frac{C_{c} m_{2}}{\bar{\gamma}_{2}}\right)^{m_{2}}{ }_{2} F_{1}\left(1+m_{1}, 1+m_{2}+n ; 2 ;-\frac{2 a \bar{\gamma}_{1}}{m_{1}}\right)\right.\right. \\
&\left.\left.+\frac{\Gamma(1+n)}{n !\left(1-m_{2}\right)_{n}}{ }_{2} F_{1}\left(1+m_{1}, 1+n ; 2 ;-\frac{2 a \bar{\gamma}_{1}}{m_{1}}\right)\right\}\right] \\
&+\frac{1}{6}{ }_{1} F_{0}\left(m_{0} ;-;-\frac{4 a \bar{\gamma}_{0}}{3 m_{0}}\right)\left[1-\left(\frac{4}{3} a \bar{\gamma}_{1}\right) \sum_{n=0}^{\infty}\left(\frac{C_{c} m_{2}}{\bar{\gamma}_{2}}\right)^{n}\left\{\frac{\Gamma\left(-m_{2}\right) \Gamma\left(1+m_{2}+n\right)}{n ! \Gamma\left(m_{2}\right)\left(1+m_{2}\right)_{n}}\left(\frac{C_{c} m_{2}}{\bar{\gamma}_{2}}\right)^{m_{2}}{ }_{2} F_{1}\left(1+m_{1}, 1+m_{2}+n ; 2 ;-\frac{4 a \bar{\gamma}_{1}}{3 m_{1}}\right)\right.\right. \\
&\left.\left.+\frac{\Gamma(1+n)}{n !\left(1-m_{2}\right)_{n}}{ }_{2} F_{1}\left(1+m_{1}, 1+n ; 2 ;-\frac{4 a \bar{\gamma}_{1}}{3 m_{1}}\right)\right\}\right] .
\end{aligned}
$$

for the Nakagami fading parameter of the r-d link, whereas Fig. $2 b$ considers the case of integer-valued fading parameter, $m_{2}=2$. For comparison purposes, the simulated ABEP and the exact ABEP expression of [7] are also shown in both sub-figures. It can be observed from these figures that the proposed expression is in excellent agreement with simulations and the exact ABEP expression of [7]. It may be mentioned here that in Fig. 2b, the graphs for simulated ABEP and the expression of [7] are plotted using the exact value $m_{2}=2$, whereas the proposed analytical expression is evaluated by setting $m_{2}=2.01$, i.e., $\delta=0.01$. The excellent match with [7] and simulations in Fig. 2b validate the effectiveness of our approach mentioned previously in footnote ${ }^{1}$ to handle integer $m_{2}$.

In Fig. 3, the proposed ABEP approximation is plotted for balanced links as well as the case of power-imbalance between the relayed links, i.e., $\bar{\gamma}_{2} \neq \bar{\gamma}_{1}$. Such an imbalance can arise for example in strong line-of-sight scenarios such that $\bar{\gamma}_{1}>\bar{\gamma}_{2}$ when relay is located closer to source than to destination and $\bar{\gamma}_{1}<\bar{\gamma}_{2}$ for relay closer to destination. Fig. 3 shows that the power imbalance is beneficial to system er- ror performance when $\bar{\gamma}_{1}<\bar{\gamma}_{2}$, whereas $\bar{\gamma}_{1}>\bar{\gamma}_{2}$ can have higher error rates than the balanced case due to relay saturation effects as also noted in [4, 8]. From Fig. 3 it can be observed that the proposed ABEP approximation provides an accurate evaluation of the ABEP for both balanced and unbalanced relayed links.

Figure 4 shows the sufficiency of using 15 terms of the infinite sums in (25) for plotting the numerical results. From the figure it can be observed that for the balanced case $\bar{\gamma}_{2}=\bar{\gamma}_{1}$ and the unbalanced case $\bar{\gamma}_{2}>\bar{\gamma}_{1}$ stable values of the approximation are obtained using only 5 terms of the infinite sums. However, the other unbalanced case $\bar{\gamma}_{1}>\bar{\gamma}_{2}$ requires 15 terms of the infinite sums. This behavior can be explained by the expression appearing in first pair of square brackets in (25) and noting that an identical explanation applies to the other two square bracket pairs in (25). For $\bar{\gamma}_{2}>\bar{\gamma}_{1}$ and $\bar{\gamma}_{2}=\bar{\gamma}_{1}$ both the factor $\left(\frac{C_{c} m_{2}}{\bar{\gamma}_{2}}\right)^{n}$ and the sum enclosed within curly braces decrease with increasing $n$ such that 5 terms of the infinite sums are sufficient to get stable and accurate values. The summand terms within the curly braces decrease with increasing $n$ due to the factorial of $n$ and 


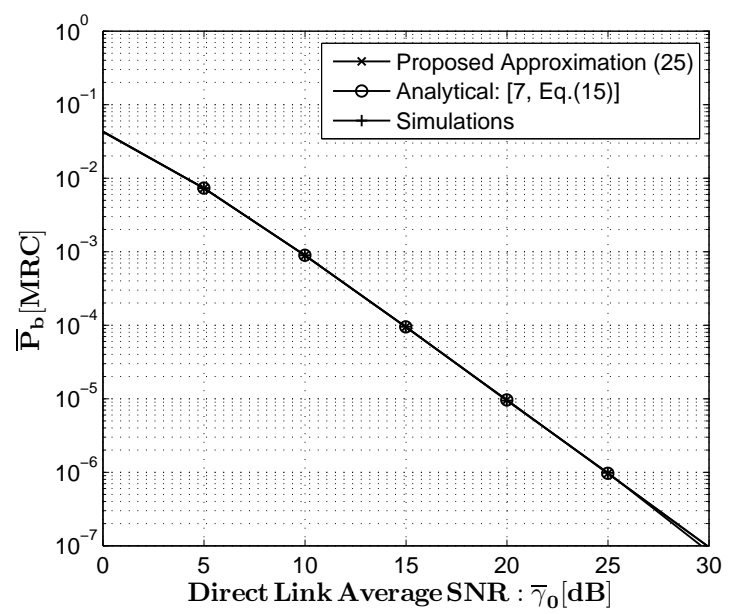

(a) Link $m$-parameters: $\left(m_{0}, m_{1}, m_{2}\right)=(1.5,2,0.5)$.

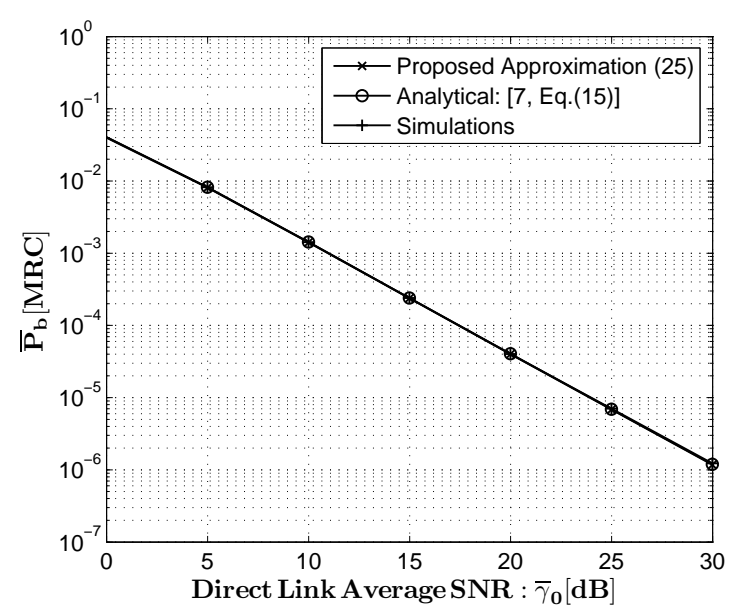

(b) Link $m$-parameters: $\left(m_{0}, m_{1}, m_{2}\right)=(0.5,1,2)$.

Fig. 2. BPSK ABEP approximation for power-balanced links, $\bar{\gamma}_{2}=\bar{\gamma}_{1}$. Furthermore, $\bar{\gamma}_{1}=5 \bar{\gamma}_{0}$.

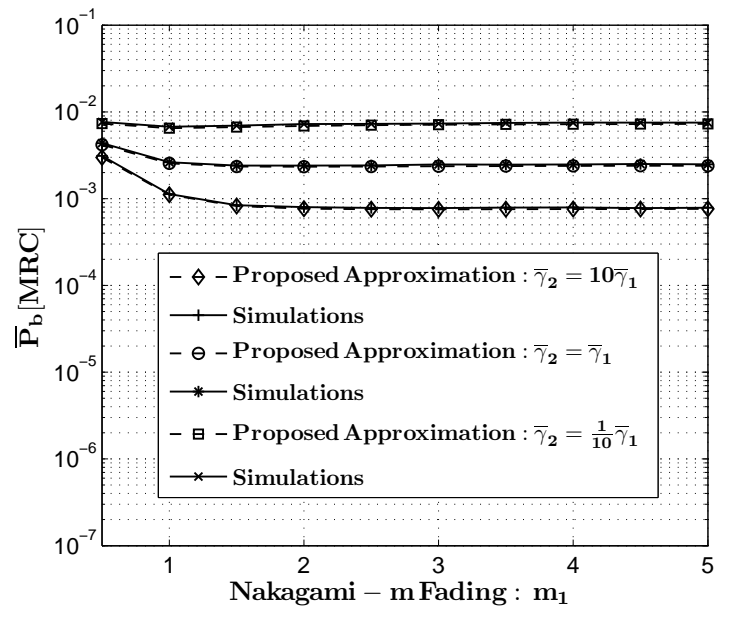

Fig. 3. BPSK ABEP approximation for balanced and unbalanced links. Link $m$-parameters are $m_{0}=m_{2}=0.5$. Link SNRs are $\bar{\gamma}_{1}=\bar{\gamma}_{0}=17 \mathrm{~dB}$.

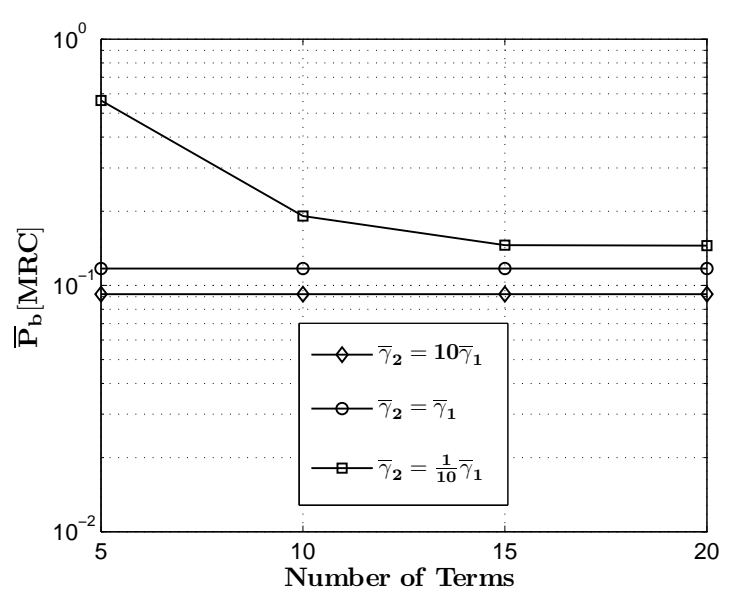

Fig. 4. Convergence of ABEP approximation for balanced and unbalanced links. Link m-parameters are $m_{0}=m_{1}=$ $m_{2}=0.5$. Link SNRs are $\bar{\gamma}_{1}=\bar{\gamma}_{0}=1 \mathrm{~dB}$.

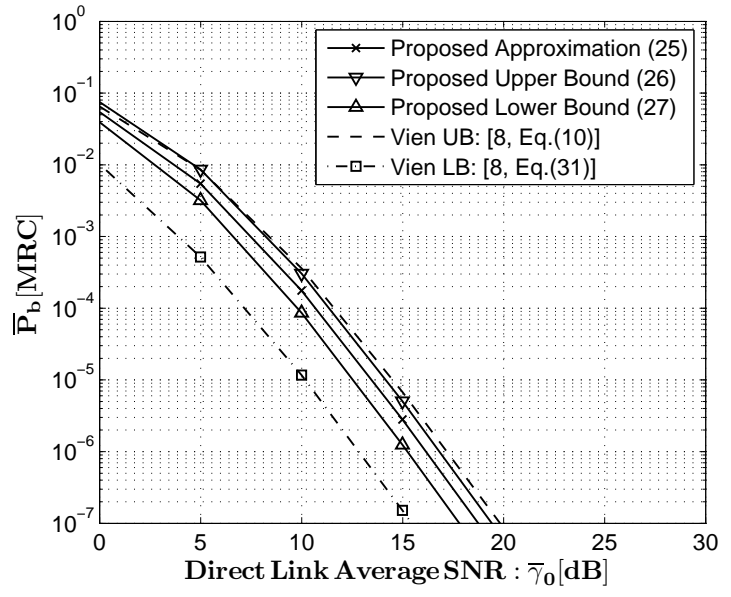

Fig. 5. Comparison of BPSK ABEP upper and lower bounds. Link $m$-parameters are $m_{0}=m_{1}=m_{2}=2$. Link SNRs are $\bar{\gamma}_{2}=5 \bar{\gamma}_{1}$ and $\bar{\gamma}_{1}=\bar{\gamma}_{0}$.

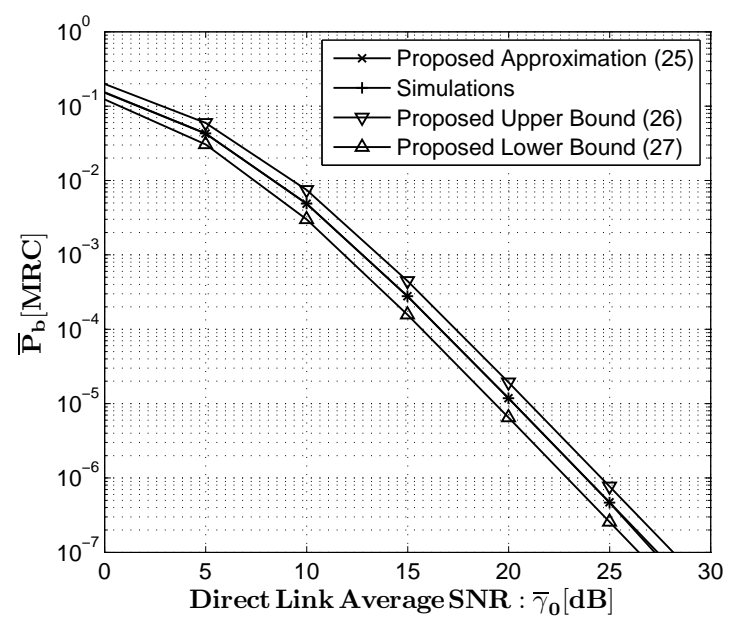

Fig. 6. BFSK ABEP approximation and bounds. Link $m$ parameters are: $\left(m_{0}, m_{1}, m_{2}\right)=(2.3,1.7,0.5)$. Link SNRs are $\bar{\gamma}_{2}=\bar{\gamma}_{1}=\bar{\gamma}_{0}$. 
the Pochhammer's symbol appearing in the denominators and $n$ appearing as second parameter of the hypergeometric functions within the curly braces. However, for the case $\bar{\gamma}_{1}>\bar{\gamma}_{2}$ and small values of $\bar{\gamma}_{1}$ such as $\bar{\gamma}_{1}=1 \mathrm{~dB}$ shown in Fig. 4 , the factor $\left(\frac{C_{c} m_{2}}{\bar{\gamma}_{2}}\right)^{n}$ grows with increasing $n$, whereas the magnitude of the sum within curly braces decreases with increasing $n$ as before. Furthermore the product of $\left(\frac{C_{c} m_{2}}{\bar{\gamma}_{2}}\right)^{n}$ and the sum within curly braces evaluates to negative quantities for a few initial values of $n$ and evaluates to positive quantities for subsequent values of $n$ such that the infinite sum converges to a positive stable value for 15 terms, as observed in Fig. 4 for the case $\bar{\gamma}_{1}>\bar{\gamma}_{2}$. Although convergence has been discussed here in the context of (25), the explanations are also applicable to the infinite sums in (26) and (27) due to similarity in the mathematical form of these expressions.

In Fig. 5, our proposed upper and lower bounds on the ABEP are plotted in comparison with the bounds derived in [8] for integer-valued $m$ parameters of the Nakagami faded links. The bounds of [8] are plotted in Fig. 5 using the exact value $m_{2}=2$, whereas the proposed bounds plotted in the same figure are obtained by setting $m_{2}=2.01$, i.e., $\delta=0.01$. It can be observed from Fig. 5 that our proposed upper bound is tighter than the upper bound of [8] at high SNRs, whereas at low SNRs both bounds have identical performance. However, our bound has the additional advantage of being much simpler to evaluate than the upper bound of [8], which requires numerical integration for evaluation. It can also be observed from Fig. 5 that our proposed lower bound is much tighter than the lower bound of [8]. This follows from the fact that the latter is based on the Harmonic/Geometric mean inequality, which loses its tightness as the power imbalance between the relayed links increases. It is pertinent to mention here that the bounds of [8] are only valid for identically distributed Nakagami faded links with the same integer value of $m$ on all links, whereas our proposed bounds do not have these restrictions.

In Fig. 6, the ABEP approximation for BFSK and its bounds are plotted for the case of $\bar{\gamma}_{2}=\bar{\gamma}_{1}=\bar{\gamma}_{0}$. The simulation ABEP is also plotted in the same figure. It can be observed from Fig. 6 that the analytical and simulation results are in excellent agreement, which validates the accuracy of our analytical expressions.

\section{Conclusion}

We have derived a simple yet accurate closed-form approximation and tight upper and lower bounds for the ABEP of BPSK and BFSK for an FG-AF relaying system, with MRC at destination, operating over independent but not necessarily identically distributed Nakagami- $m$ faded channels. The proposed analytical expressions are in good agreement with simulations and are also shown to perform better than similar expressions previously proposed in the literature.

\section{References}

[1] LANEMAN, J. N., TSE, D. N. C., WORNELL, G. W. Cooperative diversity in wireless networks: efficient protocols and outage behaviour. IEEE Transactions on Information Theory, 2004, vol. 50, no. 12, p. 3062-3080. DOI: 10.1109/TIT.2004.838089

[2] PABST, R., WALKE, B. H. et al. Relay-based deployment concepts for wireless and mobile broadband radio. IEEE Communications Magazine, 2004, vol. 42, no. 9, p. 80-89. DOI: 10.1109/MCOM.2004.1336724

[3] SURAWEERA, H. A., MICHALOPOUlOS, D. S., KARAGIANNIDIS, G. K. Performance of distributed diversity systems with a single amplify-and-forward relay. IEEE Transactions on Vehicular Technology, 2009, vol. 58, no. 5, p. 2603-2608. DOI: 10.1109/TVT.2008.2007798

[4] HASNA, M. O., ALOUINI, M.-S. A performance study of dualhop transmissions with fixed gain relays. IEEE Transactions on Wireless Communications, 2004, vol. 3, no. 6, p. 1963-1968. DOI: 10.1109/TWC.2004.837470

[5] SURAWEERA, H., KARAGIANNIDIS, G. K. Closed-form error analysis of the non-identical Nakagami-m relay fading channel. IEEE Communications Letters, 2008, vol. 12, no. 4, p. 259-261. DOI: 10.1109/LCOMM.2008.071922

[6] XIA, M., XING, C., WU, Y.-C., et al. Exact performance analysis of dual-hop semi-blind AF relaying over arbitrary Nakagami-m fading channels. IEEE Transactions on Wireless Communications, 2011, vol. 10, no. 10, p. 3449-3459. DOI: 10.1109/TWC.2011.080311.102267

[7] SHIN, H., SONG, J. B. MRC analysis of cooperative diversity with fixed-gain relays in Nakagami-m fading channels. IEEE Transactions on Wireless Communications, 2008, vol. 7, no. 6, p. 2069-2074. DOI: 10.1109/TWC.2008.070812

[8] VIEN, N. H., NGUYEN, H. H. Performance of fixed-gain amplify-and-forward relaying with MRC. IEEE Transactions on Vehicular Technology, 2010, vol. 59, no. 3, p. 1544-1552. DOI: 10.1109/TVT.2010.2040755

[9] DATTA, S. N., CHAKRABARTI, S., ROY, R. Comprehensive error performance analysis of distributed selection combining with multi-antenna amplify-and-forward relay over Nakagami-m fading channels. IET Electronics Letters, 2010, vol. 46, no. 22, p. 1-2. DOI: 10.1049/el.2010.2155

[10] KIM, Y. G., BEAULIEU, N. C. Exact closed-form solutions for the BEP of decode-and-forward cooperative systems in Nakagami-m fading channels. IEEE Transactions on Communications, 2011, vol. 59, no. 9, p. 2355-2361. DOI: 10.1109/TCOMM.2011.062311.100040

[11] CHANDRA, A., BOSE, C. BER of differentially detected $\frac{\pi}{4}$-DQPSK with selection combining in Nakagami-m fading. International Journal of Wireless Information Networks, 2010, vol. 17, no. 1, p. 54-63. DOI: $10.1007 / \mathrm{s} 10776-010-0115-\mathrm{Z}$

[12] GOLDSMith, A. Wireless Communications. 1st ed. Cambridge: Cambridge University Press, 2005. ISBN: 9780521837163

[13] GRADSHTEYN, I. S., RYZHIK, I. M. Table of Integrals, Series and Products. 7th ed. Burlington: Academic Press, 2007. ISBN: 978-0123736376

[14] FARHADI, G., BEAULIEU, N. C. A general framework for symbol error probability analysis of wireless system and its application in amplify-and-forward multihop relaying. IEEE Transactions on Vehicular Technology, 2010, vol. 59, no. 3, p. 1505-1511. DOI: $10.1109 /$ TVT.2009.2037642

[15] DATTA, S. N., BOSE, C., CHANDRA, A. Unified analysis of error performance for binary signalling over Rayleigh fading channels. IET Electronics Letters, 2007, vol. 43, no. 17, p. 934-935. DOI: 10.1049/el.20070732 
[16] ABRAMOWITZ, M., STEGUN, I. A. Handbook of Mathematical Functions with Formulas, Graphs and Mathematical Tables. 10th ed. New York: Dover, 1972.

[17] MOLISCH, A. F. Wireless Communications. 2nd ed. Chichester: Wiley-IEEE Press, 2010. ISBN: 978-0-470-74186-3

[18] CHIANI, M., DARDARI, D., SIMON, M. K. New exponential bounds and approximations for the computation of error probability in fading channels. IEEE Transactions on Wireless Communications, 2003, vol. 2, no. 4, p. 840-845. DOI: 10.1109/TWC.2003.814350

[19] FU, H., WU, M. W., KAM, P. Y. Lower bound on averages of the product of L Gaussian Q-functions over Nakagami-m fading. In Proceedings 77th IEEE Vehicular Technology Conference (VTC Spring). Dresden, 2013, p. 1-5. DOI: 10.1109/VTCSpring.2013.6692623

[20] OLABIYI, O., ANNAMALAI, A. Invertible exponential-type approximations for gaussian probability integral $\mathrm{Q}(\mathrm{x})$ with applications. IEEE Wireless Communications Letters, 2012, vol. 1, no. 5, p. 544-547. DOI: 10.1109/WCL.2012.080112.120232

[21] BREMAUD, P. An Introduction to Probabilistic Modeling. 3rd ed. New York: Springer-Verlag, 1988. ISBN: 3540964606

[22] TRIGUI, I., AFFES, S., YACOUB, M. D. Exact error analysis of dual-hop fixed-gain AF relaying over arbitrary Nakagami-m fading. In Proceedings IEEE Vehicular Technology Conference (VTC Fall). Quebec City, 2012, p. 1-5. DOI: 10.1109/VTCFall.2012.6399349

[23] WEISSTEIN, E. W. Modified Bessel Function of the Second Kind. [Online] Available at: http://functions.wolfram.com/BesselTypeFunctions/BesselK/26/01/02/

\section{About the Authors...}

Sajid H. ALVI received his MSc and MPhil degrees in Electronics from Quaid-I-Azam University Islamabad, Pakistan in 2001 and 2006, respectively. Between 2001 and 2004, he was a faculty member at College of Electrical and Mechanical engineering, NUST, Islamabad, Pakistan. Since 2006 he is serving as a faculty member at COMSATS Institute of Information Technology (CIIT), Islamabad, Pakistan, where he is currently an assistant professor at the Department of Physics and pursuing his $\mathrm{PhD}$ at the Electrical Engineering Department of the same institute. Sajid Alvi's research interests are in cooperative communications and signal processing.

Shurjeel WYNE received his PhD from Lund University, Sweden in 2009. Between 2009 and 2010, he was a PostDoctoral Research Fellow funded by the High-Speed Wireless Center at Lund University. Since 2010 he holds an Assistant Professorship at the Department of Electrical Engineering at CIIT, Islamabad, Pakistan. Dr. Wyne is a Co-recipient of the best paper award of the Antennas and Propagation Track in the IEEE 77th Vehicular Technology Conference (VTC2013-Spring). His research interests are in wireless channel measurements and modeling, $60 \mathrm{GHz}$ Communications, cooperative relay networks, and Multi-antenna systems. 